\title{
Complete opioid transition to sublingual Buprenorphine after abdominal surgery is associated with significant reductions in opioid requirements, but not reduction
} in hospital length of stay: a retrospective cohort study

Charlotte Heldreich', Sameer Ganatra², Zheng Lim¹ , Ilonka Meyer ${ }^{1}$, Raymond Hu', Laurence Weinberg ${ }^{1}$ and Chong O. Tan ${ }^{1 *}$

\begin{abstract}
Background: The use of sublingual buprenorphine (SLBup) for acute pain after major abdominal surgery may offer the potential advantages of unique analgesic properties and more reliable absorption during resolving ileus. We hypothesized that complete opioid transition to SLBup rather than oral oxycodone (OOxy) in the early postoperative period after major abdominal surgery would reduce hospital length of stay, and acute pain and total OMEDD (Oral Morphine Equivalent Daily Dose) requirements in the first $24 \mathrm{~h}$ from post-parenteral opioid transition.

Methods: We reviewed 146 patients who had undergone elective and emergency abdominal surgery under our quaternary referral centre's Upper Gastro-Intestinal and Colo-Rectal Surgical Units 6 months before and after the introduction of complete postoperative transition to sublingual buprenorphine, rather than oral oxycodone, in July 2017. Our primary endpoint was 24-hourly post-transition OMEDDs; secondary endpoints were 24-hourly post-transition Mean NRS-11 pain scores on movement (POM) and length of hospital stay (LOS). Univariate analysis and linear multivariate regression analyses were used to quantify effect size and identify surgical, patient \& other analgesic factors associated with these outcome measures.
\end{abstract}

Results: Patients transitioning to SLBup had reduced 24-hourly post-transition OMEDD requirements on postoperative day 2 (POD) (26 mg less, $p=0.04$ ) and NRS-11 POM at POD1 (0.7 NRS-11 units less, $p=0.01$ ). When adjusting for patient, surgical and special analgesic factors, SLBup was associated with a similar reduction in OMEDDs (Unstandardised beta-coefficient $-26 \mathrm{mg}, p=0.0001)$, but not NRS-11 POM ( $p=0.47$ ) or hospital LOS ( $p=0.16)$.

Conclusions: Our change of practice from use of OOxy to SLBup as primary transition opioid from patient-controlled analgesia delivered full opioid agonists was associated with a clinically significant decrease in 24-hourly post-parenteral opioid transition OMEDDs and improved NRS-11 POM, but without an association with hospital LOS after major

*Correspondence: drchongtan@gmail.com

1 Anaesthesia, Pain and Perioperative Medicine, Austin Hospital, Level 2 Austin Towers, 145 Studley Rd Heidelberg, Melbourne, VIC 3084, Australia

Full list of author information is available at the end of the article permits use, sharing, adaptation, distribution and reproduction in any medium or format, as long as you give appropriate credit to the original author(s) and the source, provide a link to the Creative Commons licence, and indicate if changes were made. The images or other third party material in this article are included in the article's Creative Commons licence, unless indicated otherwise in a credit line to the material. If material is not included in the article's Creative Commons licence and your intended use is not permitted by statutory regulation or exceeds the permitted use, you will need to obtain permission directly from the copyright holder. To view a copy of this licence, visit http://creativecommons.org/licenses/by/4.0/. The Creative Commons Public Domain Dedication waiver (http://creativeco mmons.org/publicdomain/zero/1.0/) applies to the data made available in this article, unless otherwise stated in a credit line to the data. 


\section{Introduction}

Major abdominal surgery carries the risk of persistent postoperative pain in $5-50 \%$ of cases [1]. Moreover, in cases of prolonged ileus, the potential for a protracted exposure to significant doses of lipid-soluble opioids may be associated with the development of opioid tolerance [2]. Concurrently, many postoperative patients may find themselves discharged on large doses of opioids for ongoing analgesia, with a proportion continuing such drug therapy because of poorly managed pain, opioid tolerance or dependence, or simply poor opioid stewardship [3]. The resulting complications are part of the $240 \%$ increase in hospitalisations and deaths in Australia related to opioid over-use since 2000 [4].

The use of buprenorphine in the treatment of acute pain has enjoyed a growing resurgence in perioperative pain management [5]. Current evidence suggests sublingual or intravenous (IV) buprenorphine has a similar analgesic and side effect profile to parenteral morphine at equipotent doses when treating acute post-surgical pain [6]. In addition, the sublingual route of administration is appealing in this context because of its ease of administration, lack of reliance on patient-controlled analgesia techniques and associated equipment, and rapid and reliable absorption. Furthermore, buprenorphine may also offer unique advantages over other commonly used opioids for postoperative pain management. It is antihyperalgesic [7], has a long duration of action, and as a less lipid-soluble agent with slower onset of action, may have a lower potential for developing dependence and tolerance. Finally, opioid antagonists have been reported to shorten ileus and hospital length of stay (LOS) after abdominal surgery [8].

Given that the application of IV opioid patient-controlled analgesia (PCA) techniques, with concurrent use of regional analgesia, neuraxial opioids or ketamine infusions are commonplace after major surgery, the use of buprenorphine is an attractive analgesic alternative. However, when transitioning postoperatively from PCA to oral analgesia in the context of major upper gastrointestinal or colorectal surgery, it is unknown whether sublingual buprenorphine (SLBup) offers improved analgesia and reduced oral morphine equivalent drug dose (OMEDD) requirements compared to orally administered oxycodone (OOxy). We primarily hypothesised that use of SLBup would be associated with significant reductions in post-parenteral OMEDD requirements compared with OOxy. Our secondary hypotheses were that use of SLBup would be associated with improved analgesia and reduced hospital LOS without any increase in opioid related critical incidents. We conducted a retrospective study to evaluate these hypotheses and inform our institution whether a prospective randomised trial is justified.

\section{Methods}

Following Human Research Ethics Committee approval (LNR/19/Austin/35), we performed a single centre retrospective cohort study of patients who underwent elective and emergency upper gastrointestinal and colorectal surgery between July 2016 - July 2019. Austin Health is a quaternary surgical service which undertakes $>150$ major abdominal resections annually. Inclusion criteria included adult patients (age $>18$ years) undergoing surgery of greater than two hours duration, requiring at least one overnight hospital received stay, and who received opioid patient-controlled analgesia (PCA) as part of their postoperative analgesic strategy. Both open and laparoscopic approaches were included. We excluded patients who had emergency surgery due to trauma, those with opioid tolerance (patients taking, for a week or longer, at least $60 \mathrm{mg}$ of morphine daily, oral oxycodone $30 \mathrm{mg} /$ day, transdermal fentanyl $25 \mathrm{mug} / \mathrm{hr}$., $8 \mathrm{mg}$ of hydromorphone, or an equianalgesic dose of another opioid), and patients who received percutaneous jejunal feeding. Given the retrospective nature of the study, the ethics committee waived the need for patient consent.

All surgeries were performed by specialist gastrointestinal surgeons and all patients underwent a standardised enhanced recovery after surgery program that included preoperative optimisation of comorbidities if required and prehabilitation, standardised intraoperative anaesthesia with goal directed therapy to optimise fluid intervention and guide the use of vasoactive medications, and a standardised postoperative care pathway, which included daily physiotherapy and acute pain service reviews. In the absence of contraindications, all patients received paracetamol and non-steroidal anti-inflammatory medications as part of a multimodal analgesic approach.

Two independent investigators extracted data from Austin Health Cerner ${ }^{\circledR}$ electronic medical records, which allows comprehensive electronic data capture and retrieval of patient health information in the perioperative setting. This study is reported in accordance with Strengthening the Reporting Of Cohort Studies 
in Surgery (STROCSS) guidelines [9]. Collected data included patient demographics, 11-point pain Numerical Rating Scores (NRS-11 scores), oral morphine equivalent daily dose (OMEDD) data and hospital length of stay.

In July 2017, the Austin Health Acute Pain Service initiated a practice change that transitioned patients from a postoperative regime of OOxy to a protocol using SLBup exclusively. Between July 2016 and July 2017 patients were transitioned to pro re nata (prn) OOxy immediaterelease from opioid PCA therapy at $50 \%$ of the equianalgesic dose and frequency relative to their opioid PCA use over the preceding $24 \mathrm{~h}$. This was done once patients required less than 150 OMEDDs by PCA in the last $24 \mathrm{~h}$ and were transitioned from nil-by-mouth to oral sips of fluid following surgical and dietetics review. Transition timing was a clinical decision based on multiple factors, including observed reduction in nasogastric (NG) fluid drainage, reduced abdominal distension, the appearance of bowel sounds or flatus, and absence of associated nausea and vomiting.

Patients receiving SLBup were transitioned from opioid PCA to SLBup at $50 \%$ of the equianalgesic dose and frequency relative to their opioid PCA use in the preceding $24 \mathrm{~h}$, with transition timing determined by the same PCA OMEDD threshold as those receiving OOxy, and clinical indicators for the resumption of oral medication.

\section{Outcomes}

Pain endpoints were assessed by taking the mean score derived from the 11-point Numerical Rating Score (NRS-11) per 24-h period on movement. Pain on movement was defined as the NRS-11 rating on attempted deep breathing and coughing. The outcome measure for our primary hypotheses was total opioid analgesics delivered in the $24 \mathrm{~h}$ post-transition, compared to the $24 \mathrm{~h}$ period pre-transition, for those transitioned to SLBup compared to OOxy, and converted to OMEDDs as per the Australian \& New Zealand College of Anaesthetists \& Faculty of Pain Medicine Official Conversion Table 2019 [10]. All opioids administered via all routes (intravenous, transdermal, oral, sublingual) from the time of the arrival to the recovery room, to the last dose of any opioid analgesic administered during the patients hospital stay, were summated and converted to OMEDDs as per the ANZCA conversion rates and included in the analysis if administered within the 24 -h pre- and post-transition time. Our secondary outcome measures were:

1. Pain improvement $24 \mathrm{~h}$ post-transition, compared to the $24 \mathrm{~h}$ period pre-transition, for those transitioned to SLBup compared to OOxy. This was quantified by both the absolute value of NRS-11 scale units in the
$24 \mathrm{~h}$ post-transition period as well as the fall in NRS11 scale units in this period as defined by

$[($ mean NRS - 11[24]pre - transition $)-($ mean NRS - 11[24] post - transition $)]$

(where NRS-11[24] = NRS-11 score over a 24-h period) Other secondary outcome measures assessed were frequency of pain or opioid-related Medical Emergency Team (MET) calls and hospital length of stay. Severe adverse pain or opioid-related outcomes were defined a priori as any of the following events that occurred in the 48-h period following OOxy or SLBup administration: i) thresholds of hypoxia or tachycardia where pain was deemed to be a causative factor, ii) reduced conscious state (sedation scores of 3 as per the Modified McIntyre Sedation Scale (Appendix 1c) [11]), and iii) a low respiratory rate ( $\leq 12$ breaths/minute).

To account for the effects of the acuity of surgical presentation and the size and location of the surgical incision on our primary and secondary outcome measures, we used arbitrary ordinal scales to stratify the magnitude of these factors (Appendix 1a and b, Table 2). We included single dose pre-operative intrathecal morphine, postoperative ketamine infusion, and regional analgesia (transversus abdominis plane analgesia; rectus sheath or wound catheter analgesia) under the category of special analgesia techniques (SAT) to account for the effects of these techniques on our outcome measures. The postoperative day at which opioid transition occurred was included in the multivariate regression model to account for the natural history of improved pain and reduced OMEDDs as patients recovered from surgery. Regardless of whether the transition was conducted before 'oral sips' of fluid was permitted by the surgical team, the duration of postoperative ileus was defined as the number of postoperative days until full ward diet was commenced.

\section{Statistical methodology}

All calculations were performed using SPSS V21 (IBM, New York, USA) statistical software and graphs were prepared with Graph Pad Prism 5 (Graph Pad Software, California, USA). OMEDD data and hospital LOS continuous data were assessed for normality by histogram frequency distribution analysis and the KolgomorovSmirnoff normality test, with corresponding parametric descriptive and inferential tests used (mean, SD, unpaired 2-tailed t-tests). Bonferroni correction was applied to univariate analyses where multiple comparisons were used for the same endpoint (POD 1-4 NRS-11 and OMEDDs, $P$-values $<0.0125$ considered statistically significant); otherwise $p<0.05$ was considered statistically significant. To examine the effect of confounding 
Table 1 Patient, surgical \& analgesic demographics

\begin{tabular}{|c|c|c|c|c|}
\hline & & OOxy $(N=82)$ & SLBup $(N=64)$ & $p$-value \\
\hline Age (years) & & $60(17)$ & $65(13)$ & 0.05 \\
\hline \multirow[t]{3}{*}{ ASA grade } & 2 & $41(50 \%)$ & $21(33 \%)$ & \multirow[t]{3}{*}{0.009} \\
\hline & 3 & $37(45 \%)$ & $36(56 \%)$ & \\
\hline & 4 & $4(5 \%)$ & $7(11 \%)$ & \\
\hline Sex (male) & & $36(44 \%)$ & $26(40 \%)$ & 0.61 \\
\hline Incisional Pain ordinal scale ${ }^{a}(1-3)$ & $\begin{array}{l}1 \\
2 \\
3\end{array}$ & $\begin{array}{l}22(27 \%) \\
11(13 \%) \\
49(60 \%)\end{array}$ & $\begin{array}{l}36(56 \%) \\
16(25 \%) \\
12(19 \%)\end{array}$ & 0.001 \\
\hline Emergency surgery & & $28(34 \%)$ & $32(50 \%)$ & 0.003 \\
\hline Acuity of Surgical Presentation ordinal scale ${ }^{b}(1-5)$ & $\begin{array}{l}1 \\
2 \\
3 \\
4 \\
5\end{array}$ & $\begin{array}{l}9(3 \%) \\
10(8 \%) \\
41(49 \%) \\
11(20 \%) \\
11(20 \%)\end{array}$ & $\begin{array}{l}2(1 \%) \\
9(8 \%) \\
24(33 \%) \\
14(26 \%) \\
15(32 \%)\end{array}$ & $<0.0001$ \\
\hline Postoperative day transitioned to Oxy or SLBup & $\begin{array}{l}1 \\
2 \\
3 \\
4 \\
>4\end{array}$ & $\begin{array}{l}29(35 \%) \\
28(34 \%) \\
13(16 \%) \\
8(10 \%) \\
4(5 \%)\end{array}$ & $\begin{array}{l}16(25 \%) \\
20(31 \%) \\
14(22 \%) \\
2(3 \%) \\
12(19 \%)\end{array}$ & $<0.0001$ \\
\hline POD at FWD & $\begin{array}{l}1 \\
2 \\
3 \\
4 \\
5 \\
6 \\
7 \\
>7\end{array}$ & $\begin{array}{l}6(8 \%) \\
26(34 \%) \\
22(29 \%) \\
14(18 \%) \\
4(5 \%) \\
3(4 \%) \\
1(1 \%) \\
0(0 \%)\end{array}$ & $\begin{array}{l}2(4 \%) \\
4(8 \%) \\
7(13 \%) \\
5(10 \%) \\
12(23 \%) \\
4(8 \%) \\
5(10 \%) \\
13(24 \%)\end{array}$ & $<0.0001$ \\
\hline Duration (Days, POD sips to POD of FWD) & & $2(1.2)$ & $3.6(2.9)$ & $<0.0001$ \\
\hline Length of hospital stay (days) & & $10(15)$ & $19(16)$ & 0.001 \\
\hline Special analgesia techniques (see text) & & $34(41 \%)$ & $25(39 \%)$ & 0.8 \\
\hline Laparoscopic vs Open Surgery (Lap \%) & & $49(60 \%)$ & $12(19 \%)$ & $<0.0001$ \\
\hline
\end{tabular}

Data presented as mean (standard deviation), mean (SD) or number (proportion)

ASA American Society of Anaesthesiologists; FWD full ward diet; OOXY oral oxycodone; POD postoperative day; SLBup sublingual buprenorphine

factors on our primary and secondary outcome measures, we used multivariate linear regression to account for the relative impact of patient factors (age, 24-h preand post-transition OMEDDs and NRS-11 pain rating assessments), SAT (regional analgesia, intrathecal opioid, ketamine infusions), emergency or elective surgery, ordinal scale of the expected degree of postoperative pain by surgery/incision type and ordinal scale of acuity of surgical presentation type (see appendices A and B) as well as post-transition opioid type. Analyses for collinearity were applied to ensure no dependence between covariates. A priori sample size was determined conservatively by the approximate requirement for 104 samples plus $n$ samples for each covariate used in our multivariate linear regression model [12], with the planned inclusion of a maximum of 13 covariates. Covariates were selected for inclusion in each model based on clinical relevance to the dependent variable of interest.

\section{Results}

Over the observation period, 167 patients underwent emergency and elective abdominal surgery under the care of the upper gastrointestinal and colorectal surgery units. One patient was excluded due to opioid tolerance, and 20 patients excluded who received epidural analgesia. A total of 146 patients were included in the final analysis, 82 (56\%) receiving OOxy and 64 (44\%) receiving SLBup. Patients transitioned to SLBup were more likely to be of higher ASA grade, more painful operation type, have received emergency surgery and more acute surgical presentation, and received open laparotomy. They were also more likely to have reached full ward diet later, and had longer length of stay. Patient, surgical and special analgesic categorical data are presented in Table 1.

\section{Outcomes}

On univariate analysis, patients transitioning to SLBup achieved clinically significant reductions in 24-hourly 


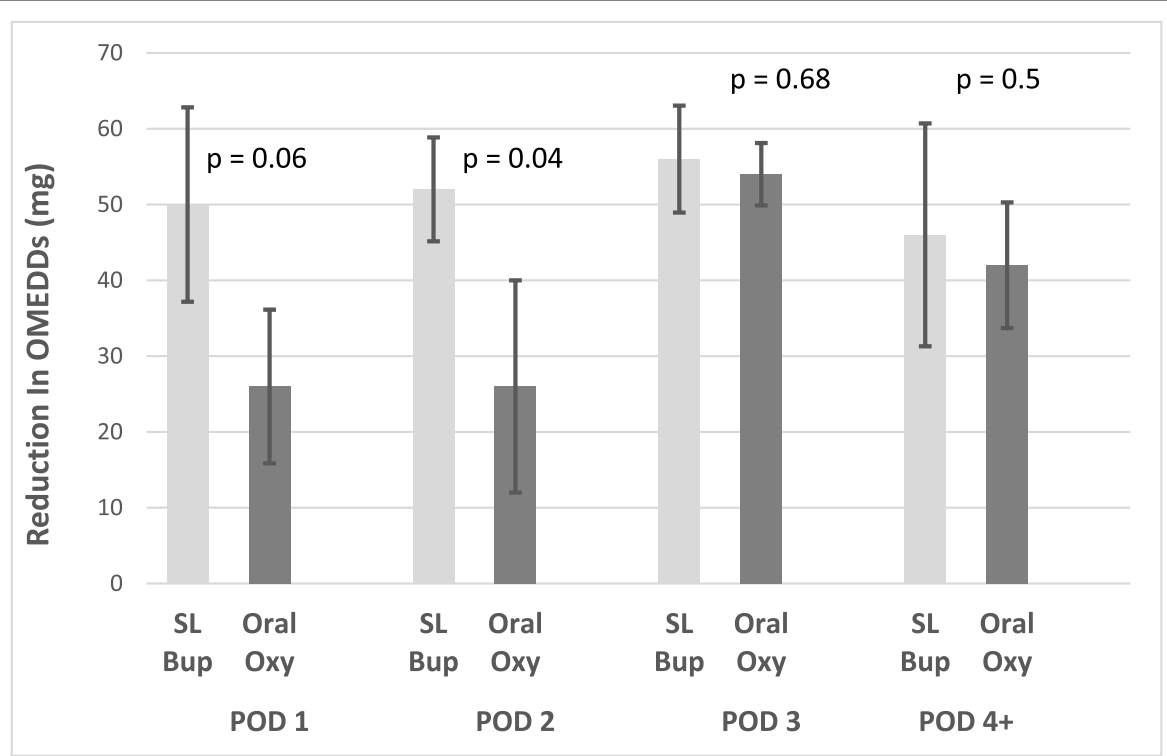

*Error bars are SEM.

Fig. 1 24-hourly post-transitional omedd reduction

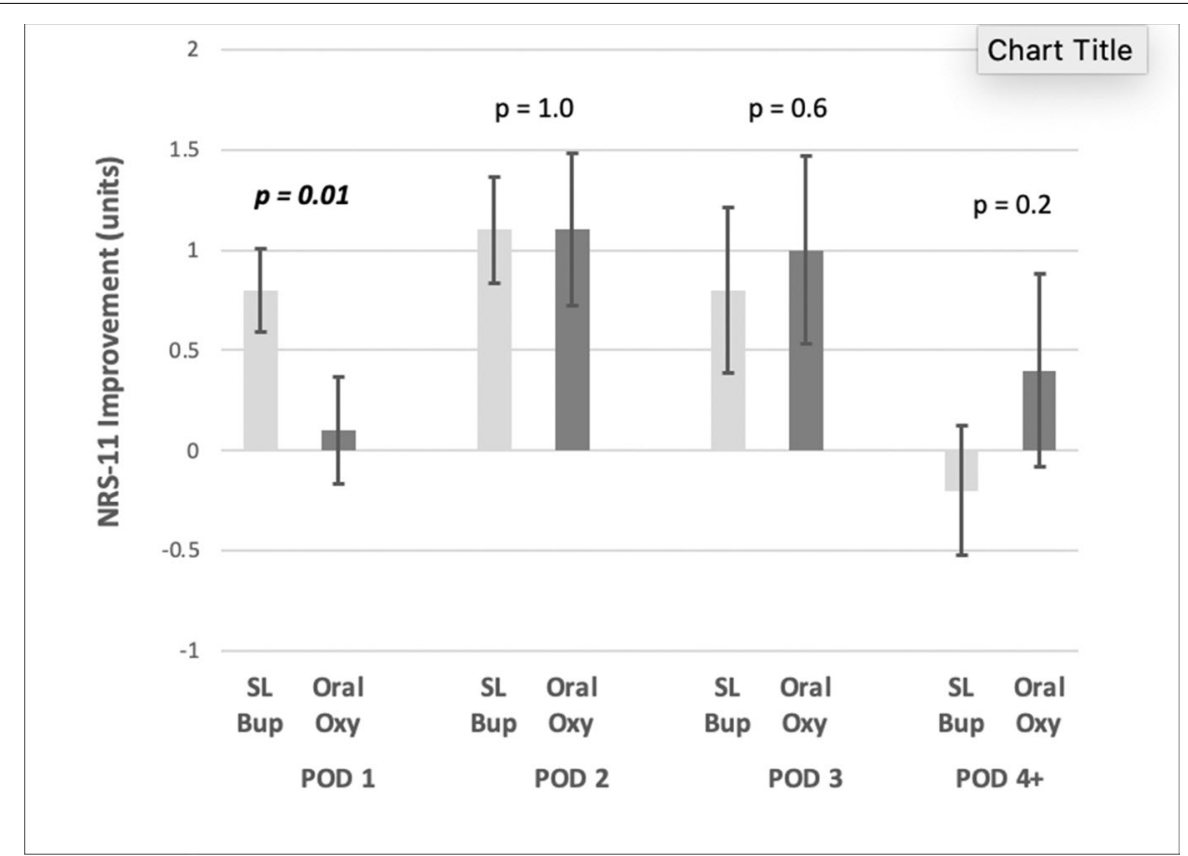

* Error bars are SEM.

Fig. 2 24-hourly post-transitional nrs-11 score improvement on movement 
Special Analgesia Technique Used

Acuity of Surgical Presentation Scale

Emergency Surgery

Mean 24-hourly pre-transition NRS-11 POM

Mean 24-hourly pre-transition OMEDDs

POD transition from parenteral opioid

Incisional Pain Rating

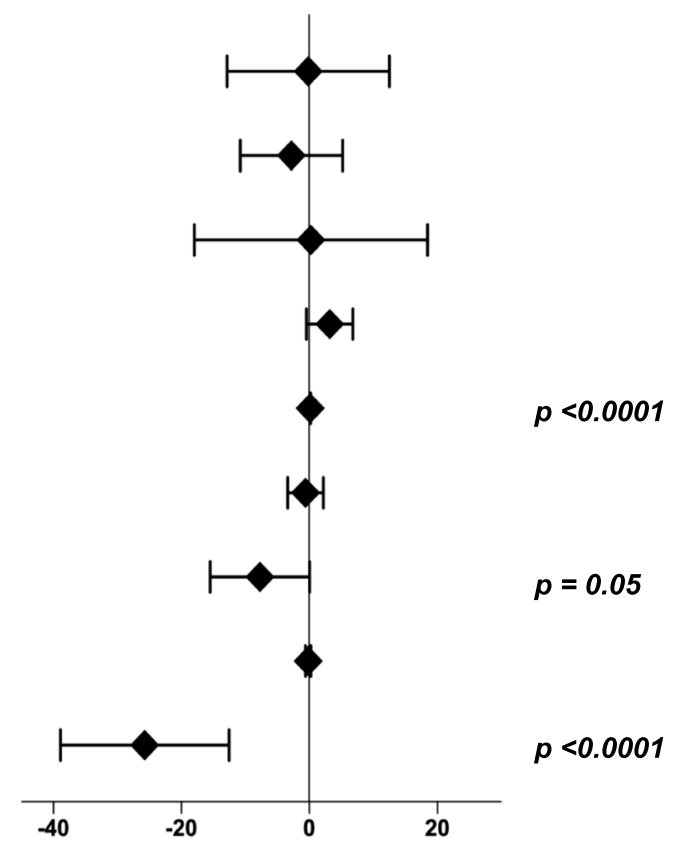

Transition to SLBup

Unstandardised Beta Coefficient

Fig. 3 Multivariate linear regression model (dependent variable: 24-hourly post-transition omedds [mg])

post-transition OMEDD requirements on postoperative day (POD) 1 and 2 (mean difference $26 \& 24 \mathrm{mg}, p=0.04$ $\& p=0.06$ respectively) that did not reach Bonferroni statistical significance, when compared against those who had transitioned to OOxy. SLBup patients did achieve statistically significant improvements in 24-hourly posttransition NRS-11 pain assessments on movement (POM) on POD 1 (mean difference 0.7 NRS-11 units, $p=0.01$ ). The 24-hourly post-transition OMEDD reductions and NRS-11 pain assessment on movement improvements are shown in Figs. 1 and 2.

On multivariate linear regression analysis with post-parenteral opioid transition 24 hourly OMEDDs as the dependent variable, patients who had received SLBup showed a statistically and clinically significant reduction in 24 hourly OMEDDs against those who had received OOxy (unstandardised betacoefficient $-26 \mathrm{mg} /$ day for those receiving SLBup, $\mathrm{p}=<0.0001$ ). This result was found after adjusting for the POD at which transition was conducted, surgical factors (incisional pain scale, emergency surgery and surgical acuity presentation scale), and other special analgesic techniques (SAT). POD of analgesic transition did not affect the total post-transition 24-hourly OMEDDs. The multivariate linear regression analysis of the covariates included in the regression model is shown in Figure 3 and Appendix 2(a), Table 3.
On multivariate linear regression analysis with postparenteral opioid transition 24-hourly NRS-11 pain improvement on movement as the dependent variable, there was no association with lower 24-hourly post-transition NRS-11 pain on movement (POM) in patients who had received SLBup over OOxy ( $p=0.32)$. This result was found after adjusting for the same surgical, pain, analgesic factors and clinical markers previously outlined. Later POD of analgesic transition worsened post-transition NRS-11 POM $(-0.04$ NRS-11 POM units per POD transition day, $p=0.02$ ). The multivariate linear regression analysis of the covariates included in this regression model is shown in Fig. 4 and Appendix 2(b), Table 4.

On multivariate linear regression analysis with hospital LOS as the dependent variable, and after adjusting for the same surgical, pain, analgesic factors and clinical markers as above, complete postoperative transition to SLBup over OOxy had no significant effect on hospital LOS $(p=0.16)$. The multivariate linear regression analysis of the covariates included in this regression model is shown in Fig. 5 and Appendix 2(c), Table 5.

Of note was a 92-year-old patient who had previously undergone an emergency infra-umbilical laparotomy and small bowel resection for bowel obstruction. The patient required administration of IV naloxone after a MET call for a respiratory rate of nine on day two postsurgery, the same day as complete transition to SLBup. 
Special Analgesia Technique Used

Acuity of Surgical Presentation Scale

Emergency Surgery

Mean 24-hourly pre-transition NRS-11 POM

Mean 24-hourly post-transition OMEDDs

POD transition from parenteral opioid

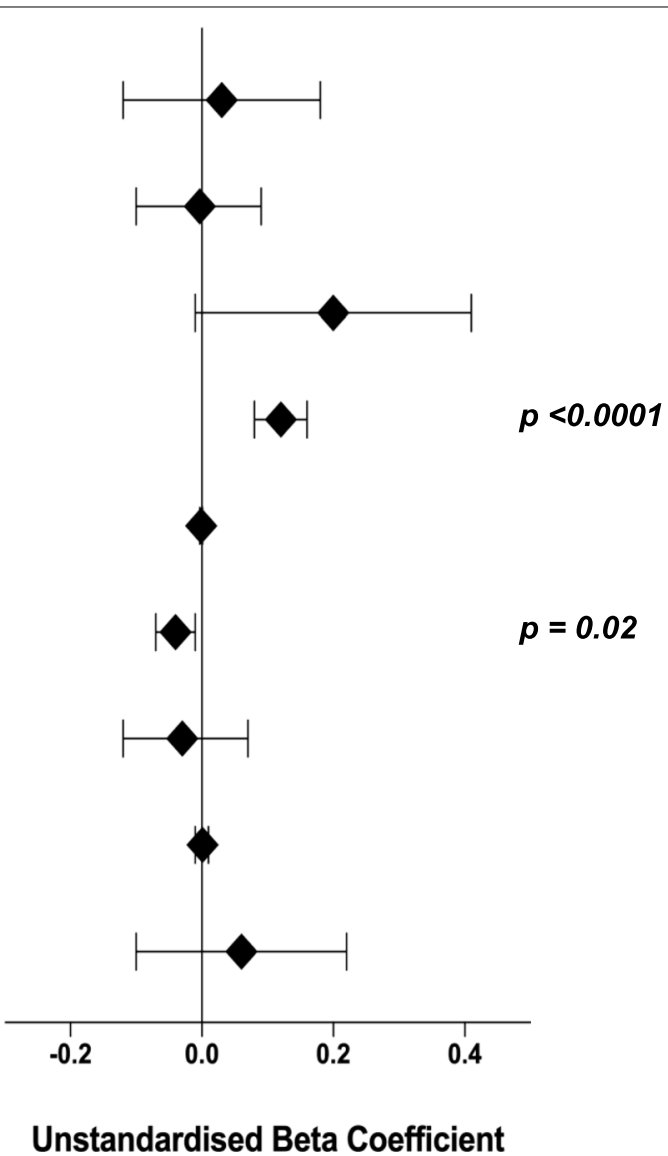

Incisional Pain Rating

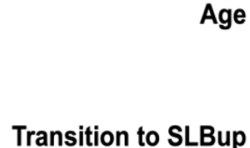

Age

Fig. 4 Multivariate linear regression model (dependent variable: 24-hourly post-transition nrs-11 pain on movement, improvement [units])

A single $200 \mu \mathrm{g}$ SLBup prn dose had been administered at $0900 \mathrm{~h}$ and second at $1900 \mathrm{~h}$, with MET threshold criteria reached for respiratory rate at $2030 \mathrm{~h} .50 \mu \mathrm{g}$ IV fentanyl had been self-administered by a PCA device since $0000 \mathrm{~h}$ on the day of transition and ceased at $0800 \mathrm{~h}$, with the patient's prior 24-hourly fentanyl PCA requirement being $300 \mu \mathrm{g}$. No other opioids had been administered, and the respiratory rate rapidly normalised to 18 breaths per minute after a single $40 \mu \mathrm{g}$ dose of naloxone. There were no other MET calls for pain or opioid-related presentations for any other study patients within 3 days of a complete transition to SLBup or OOxy.

\section{Discussion}

We performed a retrospective cohort study of patients undergoing major abdominal surgery and found that patients receiving SLBup had significantly less 24-hourly OMEDD requirements with improvement in 24-hourly analgesia on movement, without any opioid-related adverse effects. Moreover, our findings are reinforced by the observation the greatest difference in OMEDD reductions were observed at POD 1 and 2 . These results on univariate analyses were found despite patients receiving complete transition to SLBup being more likely to have surgical factors predisposing them to greater postoperative pain and OMEDD requirements.

Buprenorphine's favourable pharmacodynamic properties have long been recognised as useful in the management of chronic pain and opioid replacement therapy. The medication has low addiction potential [13], has anti-hyperalgesic [7] and anti-neuropathic [14] properties and is purported to have a lower incident rate of respiratory depression compared with other opioid classes [15]. Initial resistance to the adoption of buprenorphine as part of routine acute pain management practice has previously been based on the doubt that, as a partial agonist, its analgesic effect was limited when compared to other full opioid agonists. Contemporary randomised data have dispelled this concern, with equivalent analgesic efficacy having been demonstrated across a range of differing acute pain contexts $[16,17]$.

More recent studies have not supported the hypothesis that there is a lower risk of respiratory depression associated with buprenorphine [6]. Indeed, the only 
Acuity of Surgical Presentation Scale

Emergency Surgery

Mean 24-hourly post-transition NRS-11 POM

Mean 24-hourly pre-transition NRS-11 POM

Mean 24-hourly post-transition OMEDDS

Mean 24-hourly pre-transition OMEDDs

POD transition from parenteral opioid

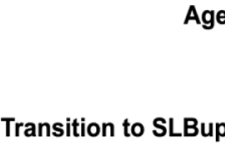

Transition to SLBup

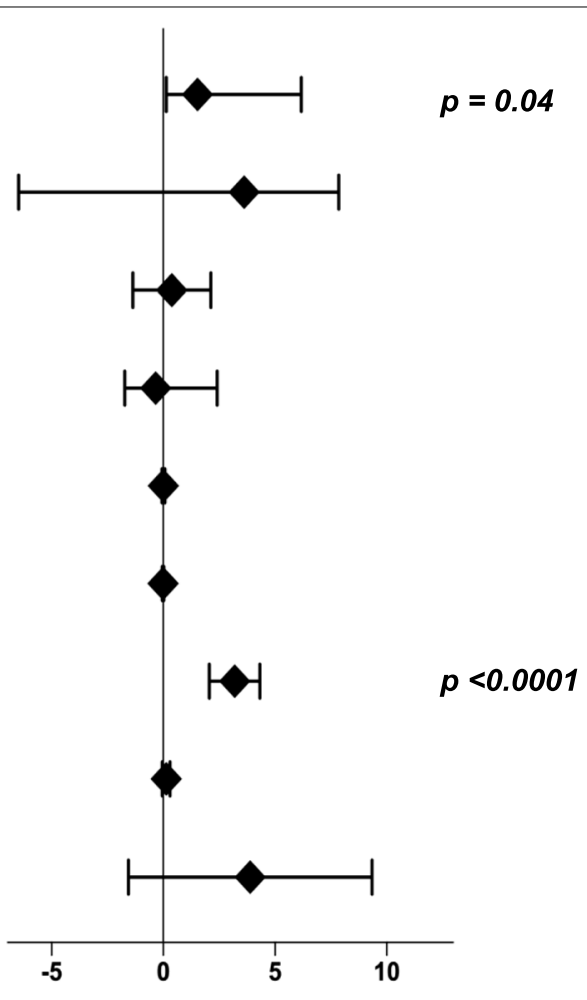

Unstandardised Beta Coefficient

Fig. 5 Multivariate linear regression model (dependent variable: hospital length of stay [days])

pain or opioid-related MET call in our study sample was in a patient receiving a very low dose of SLBup, albeit in a patient whose advanced age was a risk factor for opioid sensitivity. Opioid antagonists have demonstrated efficacy in reducing postoperative ileus time [18]. As a partial opioid agonist, buprenorphine may offer similar benefits; however, any effect of buprenorphine over oxycodone on shortening ileus time did not affect hospital LOS in our study. Although none of our results exhibits collinearity that would suggest interdependence within the covariates we analysed, our inpatient pain service's protocol of attempting opioid transition only when 24-hourly PCA OMEDD use is $<150 \mathrm{mg}$ implies that pain-related surgical or patient factors may account for a later opioid transition.

Our findings imply that SLBup is an efficacious analgesic in the context of postoperative pain management after major abdominal surgery. On univariate analysis, although OMEDD reduction when transitioning from IV opioid PCA to SLBup compared to OOxy did not reach Bonferroni statistical significance on POD 1 and 2, a clinically significant trend was displayed
(Fig. 1). Indeed, SLBup patients achieved Bonferronicorrected statistically significant improvements in 24-hourly post-transition NRS-11 pain assessments on movement on POD 1 (Fig. 2). Moreover, on multivariate analysis patients receiving SLBup showed a statistically and clinically significant reductions in 24 hourly OMEDDs compared to OOxy, reaffirming that compared to OOxy, SLBup may confer significant analgesic clinical benefits, without associated harm. Multivariate analysis did not support an association of SLBup with reduction in NRS-11 POM however, suggesting that surgical and patient factors mitigate any additional analgesic benefit of SLBup in the context of lower OMEDD requirements.

Our study is limited by several factors. As a retrospective cohort study, our findings should be considered associative and hypothesis-forming, with prospective randomised data required to draw causative conclusions. The retrospective nature of our data collection introduces many potential sources of bias and error. Our use of arbitrary incisional pain rating and acuity of surgical presentation ordinal scales is unvalidated. 
However, to our knowledge, no equivalent validated scales exist in the literature, and these scales were required to account for the effect that clinically important covariates may have on our outcomes of interest. While we have demonstrated an association between clinically important acute pain and opioid requirements, other clinically important secondary patient-centric outcomes, including postoperative complications, inpatient/post-discharge mortality, persistent postoperative pain and long-term opioid use, were not assessed. While our use of multivariate linear regression falls within accepted recommendations for the number of covariates to avoid overfitting in our modelling [19], the relatively small sample size may still lead to Type 1 or Type 2 errors in the results. We limited the maximum number of covariates included in our regression model to 9 to minimise overfitting; however, the nature of the most clinically relevant covariates for each outcome measure resulted in slightly different covariates analysed between models. We specifically did not analyse for opioid side effects of nausea and vomiting in our cohort, as these endpoints would be significantly confounded by postoperative surgical ileus and the intraabdominal nature of the surgery in our patient sample.

\section{Conclusions}

In a retrospective cohort study of 146 patients undergoing major abdominal surgery, when transitioning from full opioid agonists delivered by PCA devices to oral oxycodone or sublingual buprenorphine, patients receiving sublingual buprenorphine experienced clinically but not statistically significant reductions in post-parenteral OMEDD requirements compared to those receiving oral oxycodone. The same patients experienced a clinically and statistically significant improvement in post-transition analgesia. After adjusting for surgical, patient and analgesic factors, use of sublingual buprenorphine was associated with reduced post-transition OMEDDs, and was not associated with inferior analgesia or a reduction in hospital length of stay. Further prospective randomised studies are justified to confirm these associations.

\section{Appendix 1}

Appendix 1a - Ordinal Incisional Pain Rating Scale

$1=$ laparoscopic or laparoscopic assisted incision.

$2=$ Mini- Laparotomy or open incision $<7 \mathrm{~cm}$.

$3=$ Laparotomy or upper abdominal incision $>7 \mathrm{~cm}$.

\section{Appendix 1b}

Table 2 Ordinal Surgical Acuity of Presentation Scale

$1=$ Elective hernia or equivalent elective abdominal surgery

2 = Gastric non-cancer surgery not qualifying for higher grade; chol-

ecystitis

$3=$ Undifferentiated abdominal pain; elective cancer surgery

$4=$ Acute gastro-intestinal obstruction

$5=$ Bowel ischaemia or perforated viscus

\section{Appendix 1c - Modified McIntyre Sedation Scale}

$0=$ Awake and alert.

"S= Patient asleep but rousable.

$1=$ Easily rousable.

$2=$ Persistently drowsy, unable to stay awake spontaneously $>10 \mathrm{~s}$.

$3=$ Difficult to rouse or unresponsive.

\section{Appendix 2}

Table 3 Multivariate Linear Regression Model (Dependent Variable: 24-hourly post-transition OMEDDS)

\begin{tabular}{|c|c|c|c|c|}
\hline & \multirow{2}{*}{$\begin{array}{l}\text { Unstandardised } \\
\text { Beta Coefficients^ }\end{array}$} & \multirow[t]{2}{*}{$P$ value } & \multicolumn{2}{|c|}{ 95\% Confidence Interval for Beta } \\
\hline & & & Lower Bound & Upper Bound \\
\hline (Constant) & 56.58 & 0.017 & 10.19 & 102.97 \\
\hline $\begin{array}{l}\text { Transition to } \\
\text { SLBup }^{b}\end{array}$ & -25.73 & $<0.0001$ & -38.90 & -12.56 \\
\hline Age & -0.15 & 0.47 & -.57 & .26 \\
\hline $\begin{array}{l}\text { Incisional pain } \\
\text { rating }\end{array}$ & -7.70 & 0.05 & -15.47 & .07 \\
\hline $\begin{array}{l}\text { POD transition } \\
\text { from parenteral } \\
\text { opioid }\end{array}$ & -0.58 & 0.69 & -3.36 & 2.20 \\
\hline $\begin{array}{l}\text { Mean 24-hourly } \\
\text { pre-transition } \\
\text { OMEDDs }\end{array}$ & 0.16 & $<0.0001$ & .09 & .23 \\
\hline $\begin{array}{l}\text { Mean 24-hourly } \\
\text { pre-transition } \\
\text { NRS-11 POM }\end{array}$ & 3.19 & 0.08 & -.415 & 6.803 \\
\hline $\begin{array}{l}\text { Emergency } \\
\text { surgery }\end{array}$ & .25 & .978 & -17.96 & 18.48 \\
\hline $\begin{array}{l}\text { Acuity of surgical } \\
\text { presentation } \\
\text { scale }\end{array}$ & -2.79 & .49 & -10.80 & 5.22 \\
\hline $\begin{array}{l}\text { Special analgesia } \\
\text { technique used }^{b}\end{array}$ & -.17 & .98 & -12.86 & 12.53 \\
\hline
\end{tabular}

\#unstandardized coefficients - Covariates ( $a, b, c$ etc) affect the dependent variable (y) by $Y=$ constant + (a) $x$ [coefficient $]+(b) \times$ [coefficient]

${ }^{b}$ signifies binary covariate - If binary factor is present, there is an increase of ( $\beta$-coefficient $x$ dependent variable unit) in the dependant variable unit value. For negative $\beta$-coefficients, the dependant variable similarly decreases 
Table 4 Multivariate Linear Regression Model (Dependent Variable: 24-hourly post-transition NRS-11 pain on movement, improvement [\%])

\begin{tabular}{|c|c|c|c|c|}
\hline & \multirow{2}{*}{$\begin{array}{l}\text { Unstandardised Beta } \\
\text { Coefficients }^{\mathrm{a}}\end{array}$} & \multirow[t]{2}{*}{$P$ value } & \multicolumn{2}{|c|}{ 95\% Confidence Interval for Beta } \\
\hline & & & Lower Bound & Upper Bound \\
\hline (Constant) & 0.28 & 0.32 & -0.27 & -0.82 \\
\hline Transition to SLBup ${ }^{b}$ & 0.06 & 0.47 & -0.10 & 0.22 \\
\hline Age & 0.001 & 0.89 & -0.01 & 0.01 \\
\hline Incisional pain rating & -0.03 & 0.59 & -0.12 & 0.07 \\
\hline P-OD transition from parenteral opioid & -0.04 & .024 & -.07 & -.01 \\
\hline Mean 24-hourly pre-transition OMEDDs (?delete) & 0.001 & .52 & -.001 & .001 \\
\hline Mean 24-hourly post-transition OMEDDs & -0.001 & .38 & -.003 & .001 \\
\hline Mean 24-hourly pre-transition NRS-11 POM & 0.12 & $<.0001$ & .08 & .16 \\
\hline Emergency surgery ${ }^{b}$ & 0.20 & 0.06 & -0.01 & 0.41 \\
\hline Acuity of surgical presentation scale & -0.003 & 0.95 & -0.10 & 0.09 \\
\hline Special analgesia technique used ${ }^{b}$ & 0.03 & 0.67 & -0.12 & 0.18 \\
\hline
\end{tabular}

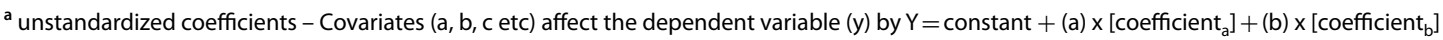

${ }^{b}$ signifies binary covariate - If binary factor is present, there is an increase of ( $\beta$-coefficient $x$ dependent variable unit) in the dependant variable unit value. For negative $\beta$-coefficients, the dependant variable similarly decreases

Table 5 Multivariate Linear Regression Model (Dependent Variable: Hospital length of stay)

\begin{tabular}{|c|c|c|c|c|}
\hline & \multirow{2}{*}{$\begin{array}{l}\text { Unstandardised Beta } \\
\text { Coefficients }^{\mathrm{a}}\end{array}$} & \multirow[t]{2}{*}{$P$ value } & \multicolumn{2}{|c|}{ 95\% Confidence Interval for Beta } \\
\hline & & & Lower Bound & Upper Bound \\
\hline (Constant) & -15.87 & .03 & -30.30 & -1.44 \\
\hline Transition to SLBup ${ }^{b}$ & 3.89 & .16 & -1.56 & 9.34 \\
\hline Age & 0.13 & .12 & -.04 & .30 \\
\hline POD transition from parenteral opioid & 3.19 & $<.0001$ & 2.06 & 4.32 \\
\hline Mean 24-hourly pre-transition OMEDDs & -0.007 & .65 & -.04 & .02 \\
\hline Mean 24-hourly post-transition OMEDDs & 0.002 & .95 & -.06 & .07 \\
\hline Mean 24-hourly pre-transition NRS-11 POM & -0.34 & .74 & -1.72 & 2.41 \\
\hline Mean 24-hourly post-transition NRS-11 POM & 0.38 & .67 & -1.36 & 2.12 \\
\hline Emergency surgery ${ }^{b}$ & 3.62 & .85 & -6.48 & 7.85 \\
\hline Acuity of surgical presentation scale & 1.53 & .041 & .13 & 6.18 \\
\hline
\end{tabular}

${ }^{a}$ unstandardized coefficients - Covariates ( $a, b, c$ etc) affect the dependent variable $(y)$ by $Y=$ constant $+(a) \times\left[\right.$ coefficient $\left._{a}\right]+(b) \times\left[c\right.$ coefficient $\left.t_{b}\right]$

${ }^{b}$ signifies binary covariate - If binary factor is present, there is an increase of ( $\beta$-coefficient $x$ dependent variable unit) in the dependant variable unit value. For negative $\beta$-coefficients, the dependant variable similarly decreases

\section{Abbreviations}

SLBup: Sublingual buprenorphine; OOxy: Oral oxycodone; POD: Postoperative day; POM: Pain on movement; NRS-11: 11-point numerical rating scale (for pain); LOS: Length of stay; PCA: Patient controlled analgesia; OMEDD: Oral Morphine Equivalent Drug Dose; MET: Medical emergency team; SAT: Special analgesia techniques; NG: Nasogastric.

\section{Acknowledgements}

Not Applicable

\section{Authors' contributions}

$\mathrm{CH}$ made substantial contributions to the conception and design of the study, the acquisition of data, and the analysis/ interpretation of data. He also drafted and revised the manuscript, and approved the final version for publication. SG made substantial contributions to the conception and design of the study, the acquisition of data, and the analysis/ interpretation of data. He also drafted and revised the manuscript, and approved the final version for publication. ZL made substantial contributions to the conception of the study, the acquisition of data, revised the manuscript for publication, and approved the final version for publication. IM made substantial contributions to the conception of the study, revised the manuscript for publication, and approved the final version for publication. $\mathrm{RH}$ made substantial contributions to the conception of the study, revised the manuscript for publication, and approved the final version for publication. LW made substantial contributions to the conception and design of the study and the analysis/ interpretation of data. He also drafted and revised the manuscript, and approved the final version for publication. CT made substantial contributions to the conception and design of the study, the acquisition of data, and the analysis/ interpretation of data. He also drafted and revised the manuscript, and approved the final version for publication. All authors read and approved the final manuscript.

\section{Funding}

No external funding was sourced for the design of the study; the collection, analysis, and interpretation of data; and in the writing of the manuscript. 


\section{Availability of data and materials}

The datasets used and/or analysed during the current study are available from the corresponding author on reasonable request. Restrictions apply to the availability of these data, as it is the Austin Hospital policy not to make outcome data for hospital patients freely available, and so will not be made publicly available. Data are however available from the authors upon reasonable request and with permission of Austin Hospital administration.

\section{Declarations}

\section{Ethics approval and consent to participate}

This study was approved by our institution's Human Research and Ethics committee (Austin Health HREC Approval \# LNR/19/Austin/35). Given the retrospective nature of the study, Austin Health ethics committee waived the needed for patient consent.

\section{Consent for publication}

Not Applicable.

\section{Competing interests}

The authors declare that they have no competing interests.

\section{Author details}

${ }^{1}$ Anaesthesia, Pain and Perioperative Medicine, Austin Hospital, Level 2 Austin Towers, 145 Studley Rd Heidelberg, Melbourne, VIC 3084, Australia. ${ }^{2}$ The Whittington Hospital, Magdala Avenue, Highgate, London N19 5NF, UK.

Received: 25 April 2021 Accepted: 17 November 2021

Published online: 21 January 2022

\section{References}

1. Richebé P, Capdevila X, Rivat C. Persistent postsurgical pain: pathophysiology and preventative pharmacologic considerations. Anesthesiology. 2018;129:590-607.

2. Schaefer CP, Tome ME, Davis TP. The opioid epidemic: a central role for the blood brain barrier in opioid analgesia and abuse. Fluids Barriers CNS. 2017;14:32.

3. Hyland SJ, Brockhaus KK, Vincent WR, Spence NZ, Lucki MM, Howkins MJ, et al. Perioperative pain management and opioid stewardship: a practical guide. Healthc Basel Switz. 2021;9:333.

4. Blanch B, Pearson S-A, Haber PS. An overview of the patterns of prescription opioid use, costs and related harms in Australia. Br J Clin Pharmacol. 2014;78:1159-66.

5. Vadivelu N, Anwar M. Buprenorphine in postoperative pain management Anesthesiol Clin. 2010:28:601-9.

6. White LD, Hodge A, Vlok R, Hurtado G, Eastern K, Melhuish TM. Efficacy and adverse effects of buprenorphine in acute pain management: systematic review and meta-analysis of randomised controlled trials. $\mathrm{Br} J$ Anaesth. 2018:120:668-78.

7. Simonnet G, Rivat C. Opioid-induced hyperalgesia: abnormal or normal pain? Neuroreport. 2003;14:1-7.

8. Vaughan-Shaw PG, Fecher IC, Harris S, Knight JS. A meta-analysis of the effectiveness of the opioid receptor antagonist alvimopan in reducing hospital length of stay and time to Gl recovery in patients enrolled in a standardized accelerated recovery program after abdominal surgery. Dis Colon Rectum. 2012;55:611-20.

9. Agha R, Abdall-Razak A, Crossley E, Dowlut N, losifidis C, Mathew G, et al. STROCSS 2019 guideline: strengthening the reporting of cohort studies in surgery. Int J Surg Lond Engl. 2019;72:156-65.

10. Ooi K. Faculty of Pain Medicine |ANZCA: official opioid dose equivalence. FPMANZCA Prof Doc 2019. http://www.anzca.edu.au/documents/anzca fpm-good-prescribing-practice-20150814.pdf. Accessed 13 Mar 2021.

11. Macintyre PE, Loadsman JA, Scott DA. Opioids, ventilation and acute pain management. Anaesth Intensive Care. 2011;39:545-58.

12. Green SB. How many subjects does it take to do a regression analysis. Multivar Behav Res. 1991:26:499-510.
13. Webster L, Gudin J, Raffa RB, Kuchera J, Rauck R, Fudin J, et al. Understanding buprenorphine for use in chronic pain: expert opinion. Pain Med. https://doi.org/10.1093/pm/pnz356.

14. Kress HG. Clinical update on the pharmacology, efficacy and safety of transdermal buprenorphine. Eur J Pain Lond Engl. 2009;13:219-30.

15. Dahan A, Yassen A, Romberg R, Sarton E, Teppema L, Olofsen E, et al. Buprenorphine induces ceiling in respiratory depression but not in analgesia. Br J Anaesth. 2006;96:627-32.

16. Jalili M, Fathi M, Moradi-Lakeh M, Zehtabchi S. Sublingual buprenorphine in acute pain management: a double-blind randomized clinical trial. Ann Emerg Med. 2012;59:276-80.

17. Oifa S, Sydoruk T, White I, Ekstein MP, Marouani N, Chazan S, et al. Effects of intravenous patient-controlled analgesia with buprenorphine and morphine alone and in combination during the first 12 postoperative hours: a randomized, double-blind, four-arm trial in adults undergoing abdominal surgery. Clin Ther. 2009;31:527-41.

18. Taguchi A, Sharma N, Saleem RM, Sessler DI, Carpenter RL, Seyedsadr M, et al. Selective postoperative inhibition of gastrointestinal opioid receptors. N Engl J Med. 2001;345:935-40.

19. Voorhis C, Morgan B. Understanding power and rules of thumb for determining sample size. Tutor Quant Methods Psychol. 2007;3.

\section{Publisher's Note}

Springer Nature remains neutral with regard to jurisdictional claims in published maps and institutional affiliations.
Ready to submit your research? Choose BMC and benefit from:

- fast, convenient online submission

- thorough peer review by experienced researchers in your field

- rapid publication on acceptance

- support for research data, including large and complex data types

- gold Open Access which fosters wider collaboration and increased citations

- maximum visibility for your research: over 100M website views per year

At BMC, research is always in progress.

Learn more biomedcentral.com/submissions 